\title{
Multi Scale Models for Flexure Deformation in Sheet Metal Forming
}

\author{
Edmondo Di Pasquale $1,2,3, a$ \\ ${ }^{1}$ SIMTECH, 2 rue AlbertSamain, F-75017 Paris, France \\ ${ }^{2}$ Univ. Lille Nord de France, F-59000 Lille, France \\ ${ }^{3}$ ENSIAME, Mont Houy, F-59313 Valenciennes, France
}

\begin{abstract}
This paper presents the application of multi scale techniques to the simulation of sheet metal forming using the one-step method. When a blank flows over the die radius, it undergoes a complex cycle of bending and unbending. First, we describe an original model for the prediction of residual plastic deformation and stresses in the blank section. This model, working on a scale about one hundred times smaller than the element size, has been implemented in SIMEX, one-step sheet metal forming simulation code. The utilisation of this multi-scale modeling technique improves greatly the accuracy of the solution. Finally, we discuss the implications of this analysis on the prediction of springback in metal forming.
\end{abstract}

\section{Introduction and state of the art}

After the first industrial applications [1]-[2], sheet metal forming simulation is nowadays part of industrial design processes. Most of the time, we use so called incremental codes for this task, where there is a Finite Element model of physical forming tools and a step-by-step simulation of their contact with the blank.

An alternative technique, one-step or inverse simulation[3], uses only the final geometry of the stamped part to predict metal deformation. This feature makes it easier to integrate this kind of simulation in the design cycle. For example, we can use the same Finite Element model for crash and metal forming simulations, making it much easier to couple the two processes and leading to much better accuracy in the prediction of performances.

This paper presents an original analytic approach to the study of the cycles of bending and unbending. This approach was developed in 2003, but it is receiving attention in this past months. Flexure bending and unbending is a process that takes place over a distance comparable to the thickness of the blank. Capturing the gradient of deformation would thus require a discretization of the order of the tenth of a millimeter, whereas typical mesh size for this kind of simulation is one hundred times larger. We have used multi scale techniques to integrate this analysis into our simulation.

Since the beginning of scientific study of metal forming [4]. Cycles of bending and unbending induced by tool geometrical features such as die radii or drawbeads to generate both a restraining force and a permanent plastic deformation.

Cycles of bending and unbending also create residual stresses, which, in turn generate springback deformations when the tools are released [5]-[8]).

In this paper, we describe the formulations of the code SIMEX as an example of one-step sheet metal forming simulations. The first version of SIMEX was released in 1994, and since 2004 it is integrated in the solution package of ESI Group. Other commercial one-step simulation codes are marketed by ALTAIR, AUTOFORM, LSTC, etc ...
This paper describes the theoretical foundation and the implementation in SIMEX of an equivalent model for the cycles of bending and unbending undergone by the blank over the die radius. This development greatly improved the accuracy of SIMEX simulation.

\section{One step metal forming simulation}

\subsection{Formulation}

The starting point of the inverse simulation is the finite element model of the stamped part. The algorithm of inverse simulation enables the user to find the position of the stamped part's nodes on the blank's initial geometry (Figure 1). This research is carried out without taking into account the history of the deformation, hence the naming of "one-step". The calculated displacements field ensures the equilibrium of the stamped part, through the calculations of the deformation (thinning, elongation) and of the constraints.

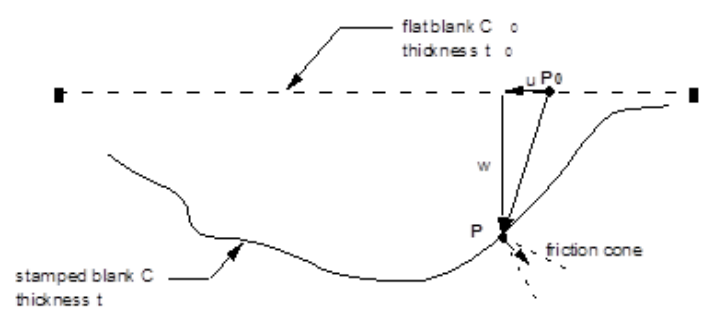

thiokness t

Figure 1: One step sheet metal forming simulation

The main feature of the inverse problem is that the FE model is built on the final shape, whereas the unknowns are distributed between the flat sheet metal (result of the calculation) and the stamped part (starting point of the calculation).

The material model implemented in SIMEX follows the plasticity laws of Henki-Mises, which are based on the following hypotheses:

The deformation's elastic component can be ignored with respect to the plastic component.

The deformation paths are radial (Figure 1), i.e. at each time $\mathrm{t}>0$

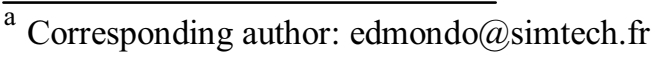




$$
\frac{\mathcal{E}_{1}(t)}{\mathcal{E}_{2}(t)}=\alpha(\text { cons } \tan t)
$$

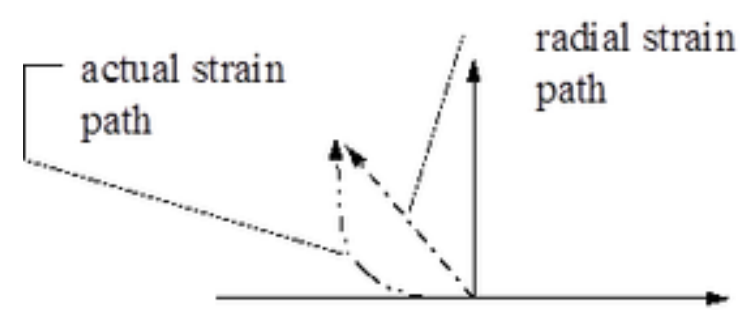

Figure 2: Radial strain path vs. actual strain path

On the basis of these hypotheses, the equations of the material flow (associative plasticity of Von Mises and Hill, [9]) can be integrated to give rise to and explicit relation between the eigenvalues of the stress and the strain tensors:

$$
\begin{aligned}
& \sigma_{1} \\
& \sigma_{2}
\end{aligned}=E_{s} P^{-1} \varepsilon_{1}
$$

Where Es is the secant modulus, i.e. the ration between $\square$ and $\square \mathrm{p}$ from the equation:

$$
\sigma=k\left(\boldsymbol{\varepsilon}_{0}+\boldsymbol{\varepsilon}_{p}\right)^{n}
$$

And $\mathrm{P}$ is a matrix, function of the chosen plasticity criterion. For the Henki-Mises criterion, we have the following $\mathrm{P}$ matrix, where $\mathrm{R}$ is the Lankford anisotropy coefficient:

$$
P^{-1}=\left[\begin{array}{cc}
\frac{(1+R)^{2}}{(1+R)(1+2 R)^{\prime}} & \frac{R(1+R)}{1+2 R^{\prime}} \\
\frac{R(1+R)}{1+2 R^{(}} & \frac{(1+R)^{2}}{(1+R)(1+2 R)^{\prime}}
\end{array}\right]
$$

As for the friction, we use Coulomb's law. Two friction-contact models are established:

Unilateral contact between the metal sheet and the punch.

Bilateral contact between metal sheet, die and blankholder.

\subsection{Modeling issues}

The most important simplification of the inverse method is therefore the search of the equilibrium in the final configuration of the process. There are two important factors which are not taken into account:

The history of deformation: The history of the contact between the sheet metal and the tool. Achieving accuracy with one-step simulation implies the elaboration and development of equivalent models, which reconstruct the history of contact and/or deformation.

In order to understand how equivalent models work in one-step sheet metal forming simulation, let us consider a simple, U-shaped forming operation. The model represents the final shape of the part, it contains 1905 nodes and 1600 quadrilateral elements. In order to define equivalent models, it must be partitioned according to the role of the different areas in the forming operations (Figure 3, which shows also the profile sections on which we shall take profiles of thinning strains). We should point out that the Finite Element model presents sharp edges for the punch and die line, whereas in reality there will be fillets. This is consistent with the modeling procedures for stress analysis and crash simulation.

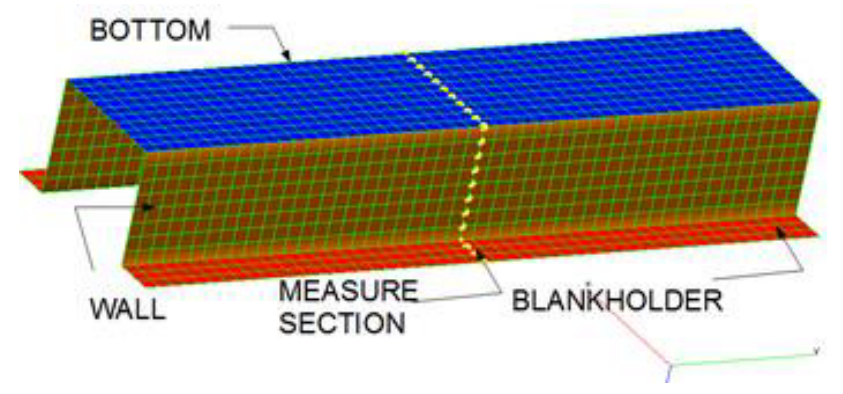

Figure 3: U-shape finite element model

In an actual punch die forming process, the advancing punch would adhere to the surface of the blank and prevent this portion of material from deformation. As one-step simulation does not take into account the history of contact, this effect is not simulated and the blank at the bottom of the U shape shows significant deformation (see the "standard bottom" thinning profile in Figure 4). Assigning a stick feature to the bottom of the punch triggers an approximate simulation of the history of contact. The deformation under the punch is negligible and the results are much more realistic.

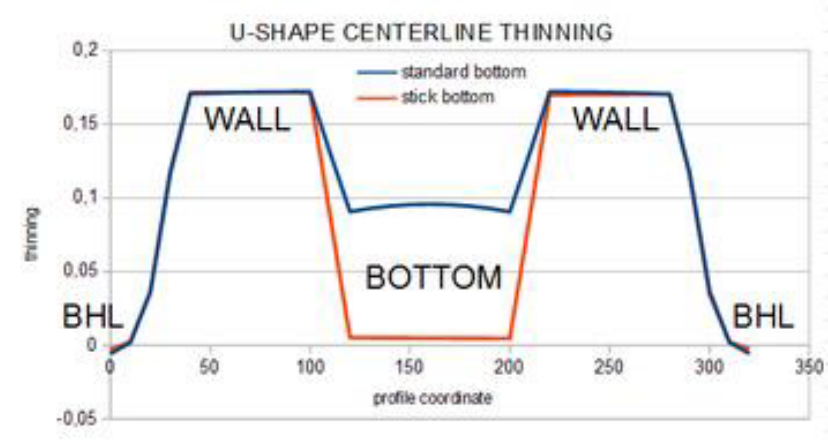

Figure 4 : Approximate simulation of history of contact using the stick feature

Taking into account the history of contact is important but not enough to assure the accuracy of the simulation. Figure 5 shows the thinning distribution (relative to the case where the bottom is modeled with stick feature. We can point out the following problems:

The wall deformation (about 17\% thinning) is caused essentially by the blankholder force through the friction coefficient. The force corresponding to this deformation is 200 tons, corresponding to $667 \mathrm{Mpa}$ of pressure for a friction coefficient of 0.2 . This is an extremely high value for the blankholder pressure with respect to industrial standards. 
The thinning distribution on the wall shows significant reduction on the extremities. In reality we would have small gradient of thinning but by no means comparable with what we see in Figure 5.

In the physical process, the process of bending/unbending over the die radius contributes greatly to the wall deformation. Among other things, it would induce a state of deformation close to a plane strain, greatly reducing the gradient of deformation along the $y$ axis of our model.

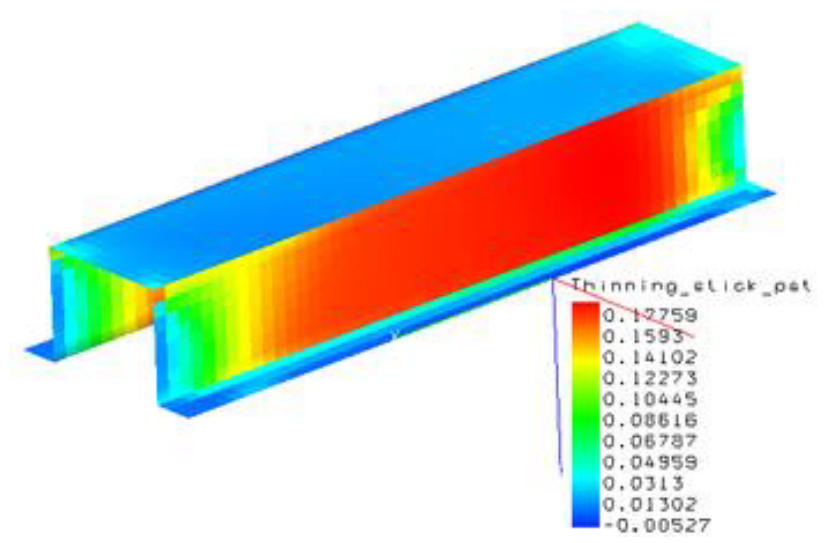

Figure 5 : Thinning distribution on U-shape part

In the following, we describe the theoretical foundation and the implementation of an equivalent model for the cycles of bending and unbending undergone by the blank over the die radius. We shall see that this improve the qualitative distribution of thinning on the U-shape part and allows for an excellent comparison between experimental and SIMEX results.

\section{Flexural deformation in metal forming}

\subsection{Description of the model}

In order to understand the physical processes involving flexure in sheet metal forming, let us consider the very simple U-shape of Figure 5. This simple shape can be obtained from two different processes: bending and forming with punch and die tooling.

In bending, the initially flat blank is bent on the four corners. Several sequences of bending are possible depending on the tooling setup. Generally speaking, the deformations of the different corners are independent on each other and the portion of materials between the bend are not affected by the deformation process. When we release the tools, each corner will undergo its own springback and recover part or the imposed deformation.

In punch and die tooling, the initially flat blank is set over a die. Prior to actual forming, a blankholder presses the blank against the die. The forming of the U-shape is obtained when a male tool (punch) pushes the blank into the die cavity. The material flowing into the die cavity undergoes a cycle of bending/unbending when it flows around the die entry radius. As a result, although the shape of the part wall may look at first sight identical to that obtained from a bending process, this material locks in both a plastic deformation and significant residual stresses. The shape of such a part after springback shows a typical curved wall.

For the case of bending and punch and die forming alike, the final shape presents four bends, i.e. areas of significant curvature. However, the deformation processes of the blank over these bends are very different.

In the case of a bending process, the deformation over the bends will be purely flexural, and can be written, as long as the radius of curvature is large with respect to the material thickness as:

$$
\boldsymbol{\varepsilon}_{x}=\frac{y}{R_{D}+h / 2}
$$

The $\mathrm{x}$ and $\mathrm{y}$ axis belonging to the curvilinear reference frame defined in Figure 6.

In the case of a form and die punch, this distribution of deformation will essentially be correct for the two bends on the punch nose or punch line. However, over the die radius, the distribution of deformation will vary greatly along the curvilinear coordinate, as it will be shown in the following.

For the sake of simplicity, we assume that the effect of friction is negligible. We also assume that the blank is in the elastic phase before entering the die radius. The blank, goes through the following process (Figure 6):

A: Unloaded elastic state. A relatively small, uniform stress and strain across the thickness can be present. The resultant force per unit width $\mathrm{T}$ will be present, for equilibrium considerations, over all the sections of the die radius.

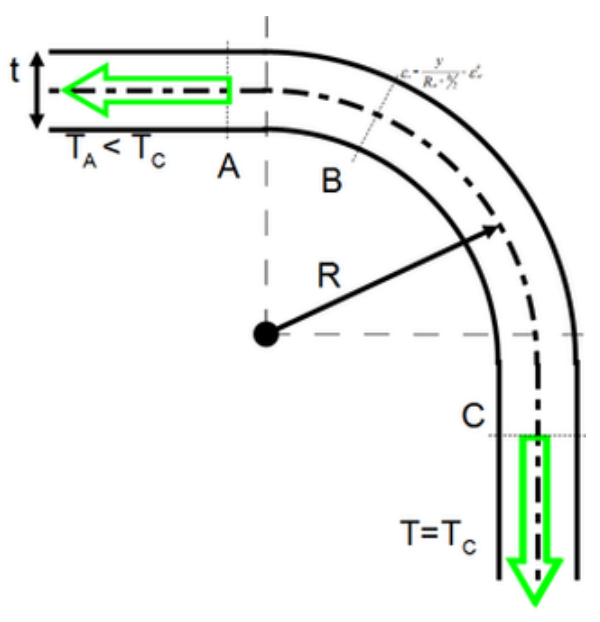

Figure 6: History of deformation over the die radius

B: After entering the die radius (classical theory of continuum mechanics suggests a distance approximately equal to the blank thickness) the section will have a curvature radius equal to $r_{D}+h / 2$. The energy required for this deformation is provided by a traction force $\mathrm{T}$ applied at the end of the die radius by the punch via the formed material. This force must be balanced, so that the deformation state of the blank will be a combination of the flexural deformation (1) and of a membrane deformation $\varepsilon_{\mathrm{M}}{ }^{\mathrm{B}}$. 


$$
\boldsymbol{\varepsilon}_{x}=\frac{y}{R_{D}+h / 2}+\boldsymbol{\varepsilon}_{M}^{B}
$$

C: At the exit of the die radius, the blank will be flat again, or in any case the residual radius of curvature will be much higher than the die radius $r_{D}$. The deformation will be again constant across the thickness, equal to a new value $\varepsilon_{\mathrm{M}}{ }^{\mathrm{C}}$.

\subsection{Analytical model of deformation}

We study the deformation of a material segment (in the sense of [10]) of blank flowing over the die radius. This treatment gives us values for the permanent plastic deformation $\varepsilon_{\mathrm{M}}{ }^{\mathrm{C}}$ and for the traction $\mathrm{T}=\mathrm{TC}$ at the end of the die bend.

We should point out that the traction force $\mathrm{T}$ may be below yield level (i.e., the ration $\mathrm{T} / \mathrm{h}$ may be less than the yield stress $\square$ y). However, plastic deformation is usually present across the whole thickness of the blank. Further, depending on the strain hardening characteristics, there is usually a gradient of stress across the thickness, leading to a springback deformation when the residual stresses are released.

Before implementation into SIMEX, we present the results of a semi-analytical analysis. In this connection, we make a few simplifying assumptions.

The first assumption is that plain sections remain plane, i.e. we can define a flexural component of the deformation equal to:

$$
\varepsilon_{f}=\frac{y}{R_{D}+h / 2}
$$

Maximum flexural deformation will take place on the upper (sup) and lower (inf) skin of the blank:

$$
\varepsilon_{f}^{\text {sup }}=\frac{h}{2\left(R_{D}+h / 2\right)}, \mathcal{E}_{f}^{\text {inf }}=-\frac{h}{2\left(R_{D}+h / 2\right)}
$$

The second assumption is that bending and unbending cause the same amount of membrane deformation across the thickness of the blank:

$$
\boldsymbol{\varepsilon}_{M}^{C}=2 \boldsymbol{\varepsilon}_{M}^{B}=\boldsymbol{\varepsilon}_{M}
$$

The third assumption is that the material hardening behavior is isotropic, i.e. the yield surface expands without shifting when the Von Mises stress increases according to the Krupkowsky-Swift law (3.1). Analysis of the effect of different hardening behaviour will be presented in a future publication.

Finally, we shall neglect the elastic component of the deformation.

Even with the above simplifying assumptions, the deformation pattern is relatively complicated.

The first results presented are relative to a typical low grade steel blank of thickness $\mathrm{h}=0.67$, drawn over a die radius $\mathrm{R}=5 \mathrm{~mm}$. The hardening curve is given by:

$$
\sigma=491\left(0.00725+\mathcal{E}_{p}\right)^{0.182}
$$

No restraining force $T_{A}$ is present at this time. Figure 7 shows the deformation profiles across the thickness for section $\mathrm{B}$ (bending loading) and C (bending unloading).

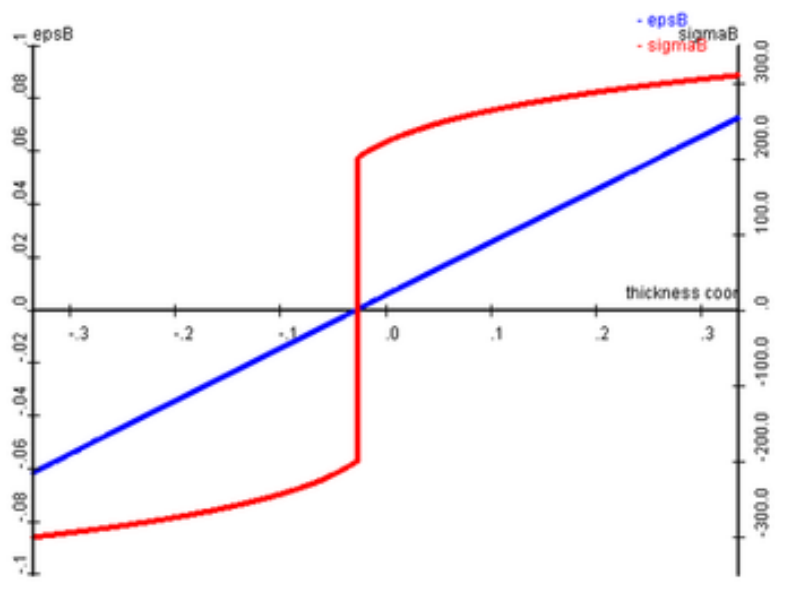

(a) Loading

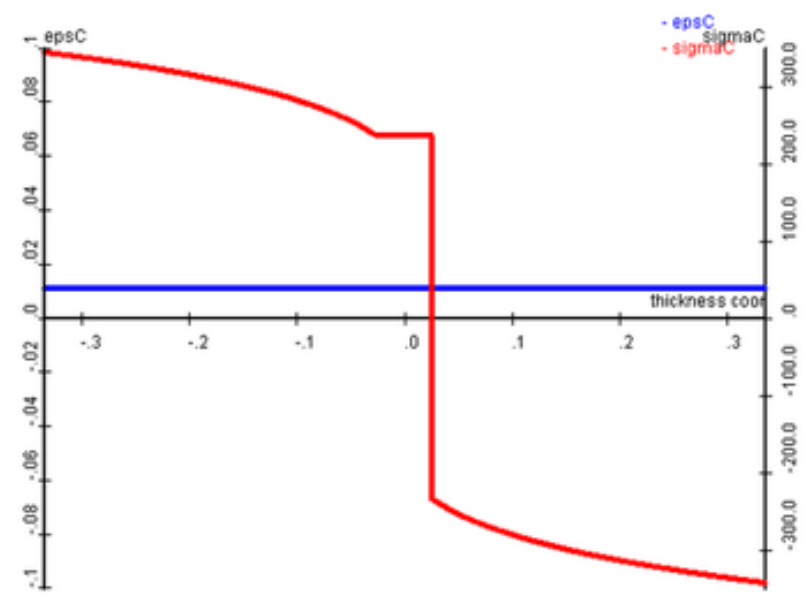

(b) Unloading

Figure 7 : Deformation and stress after bending

After the bending loading (section B) the fibers of the material are in traction above the neutral fiber $\mathrm{y}_{0}$, in compression below the neutral fiber. The value of $y_{0}$ is given by:

$$
y_{0}=\varepsilon_{M}^{B} R_{h}=\frac{\varepsilon_{M}^{B}}{2} R_{h}
$$

where

$$
R_{h}=R \quad+\frac{h}{2}
$$

Fiber stress will be above $\sigma_{\mathrm{y}}$ in the extended fibers and below $-\sigma_{\mathrm{y}}$ in the compressed fibers.

During the unbending process, the deformation will be brought to a constant value $\varepsilon_{\mathrm{M}}$, but the different fibers will follow different paths (Figure 8)

For $\mathrm{y}<-\mathrm{y}_{0}$ (lower fibers) the strain will increase to $\varepsilon_{\mathrm{M}}$ while the stress will revert above $\sigma_{\mathrm{y}}$. 
For $\mathrm{y}>\mathrm{y}_{0}$ (upper fibers) the strain will decrease to $\varepsilon_{\mathrm{M}}$ while the stress will revert below $-\sigma_{\mathrm{y}}$.

Finally, for $-\mathrm{y}_{0}<\mathrm{y}<\mathrm{y}_{0}$, the strain will increase to $\varepsilon_{\mathrm{M}}$ while the stress keeps the constant value corresponding to this plastic deformation.

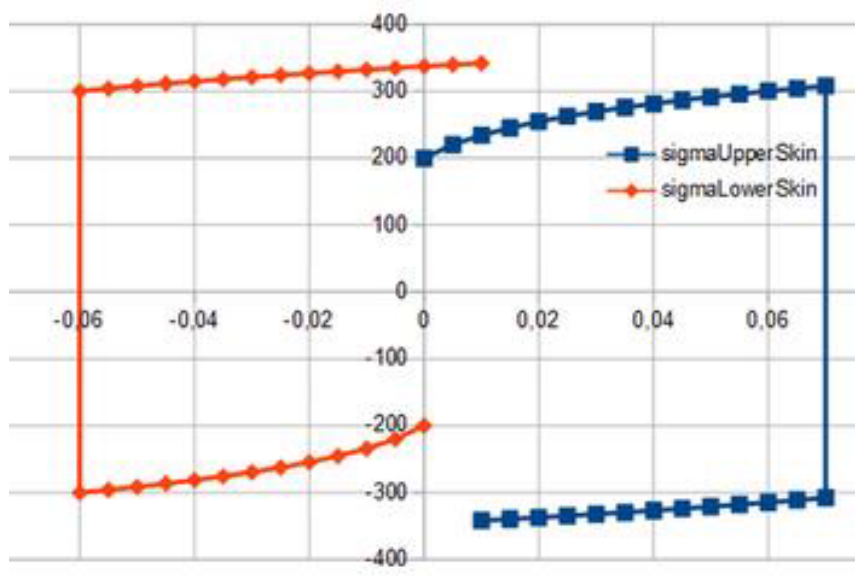

Figure 8: Stress-strain path of upper and lower skin

Finally, we can point out that the stress distribution at the end of the die radius (point $\mathrm{C}$ ) gives rise to a moment $\mathrm{M}_{\mathrm{C}}$, defined as:

$$
M_{C}=\int_{-h / 2}^{h / 2} y \sigma_{x}^{C} d y
$$

Our model predicts the restraining (traction) force $\mathrm{T}$ and the residual moment $\mathrm{M}$. We present the results for this two quantities, normalized with respect to the values corresponding to a totally plastified section:

$$
\begin{aligned}
& T_{\text {norm }}=\frac{T}{\sigma_{y} h} \\
& M_{\text {norm }}=\frac{M}{\sigma_{y} h^{2} / 4}
\end{aligned}
$$

We can now analyze the behavior of the metal sheet going through the die radius. It will be determined by the die geometry, modeled via the flexural strain $\square \mathrm{f}$, defined as:

$$
\varepsilon_{f}=\frac{h}{2 R_{h}}
$$

We analyze here the effect of the material properties, by comparing the behavior of a ductile material (low grade steel) with a perfectly plastic material.

Permanent deformation after die radius has a very similar pattern for different materials as a function of flexural strain (Figure 9).

Traction force, on the other hand, is significantly affected by material hardening. In both cases, value of traction force is very small for small flexural strains, but it increases much more rapidly in the presence of significant hardening, to a value of about $40 \%$ of $\mathrm{T}_{\text {norm }}$ (Figure 10).

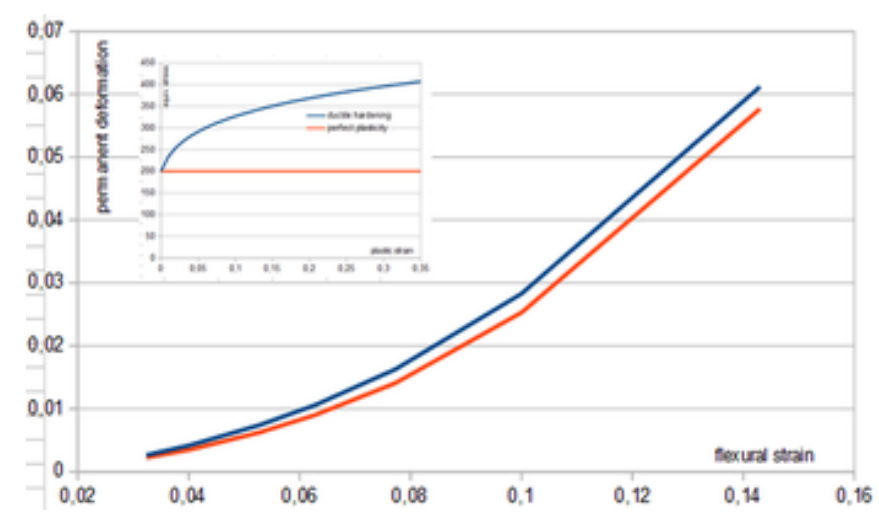

Figure 9 : permanent deformation vs. flexural strain

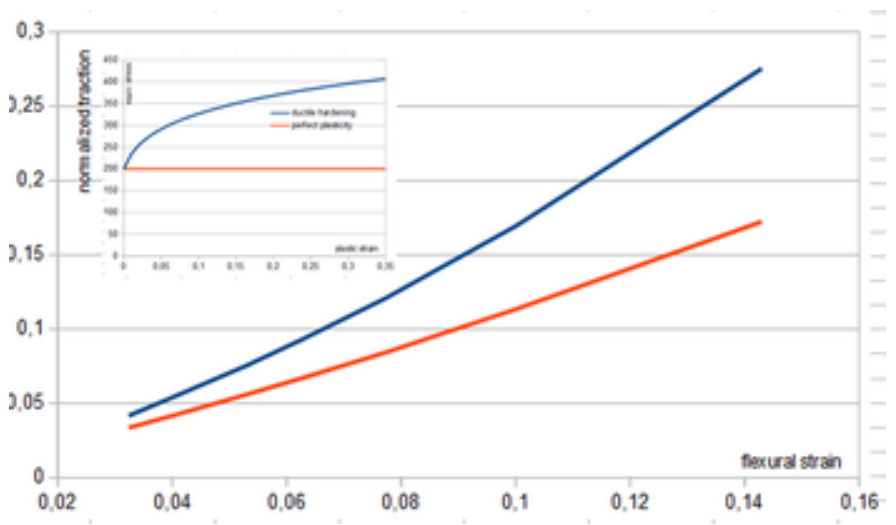

Figure 10 : traction force vs. flexural strain

Material hardening affects greatly also the resultant moment of residual stresses (Figure 11).

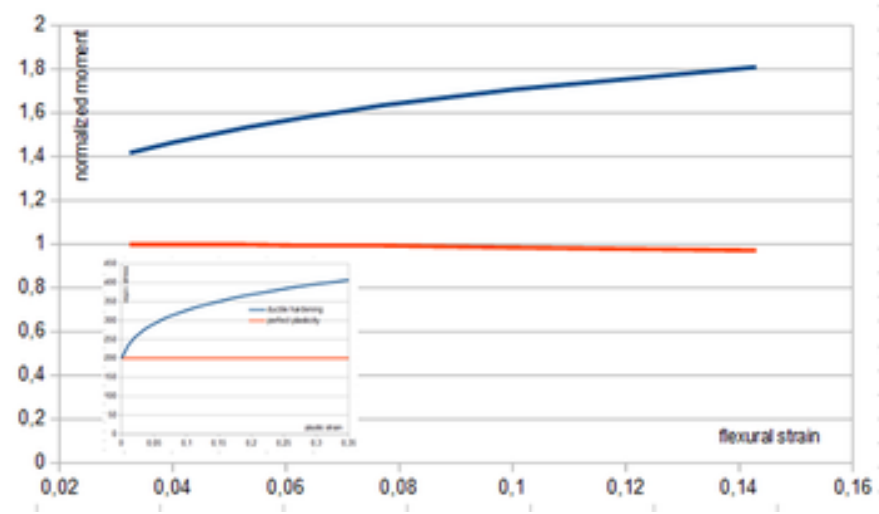

Figure 11 : residual moment vs. flexural strain

\section{Application to industrial part}

The above described methodology has been imbedded in the "bending" boundary condition of SIMEX code. Even though the mesh size is roughly the order of magnitude of the die radius, this boundary condition models the bending/unbending cycle which takes place on a much lower scale.

The French RNTL MACARENA project addressed the general issue of coupling crash computation with metal forming simulation. SimTech was a partner in 
MACARENA alongside, among others, PCA Peugeot Citroen and RENAULT.

One of the problems addressed within MACARENA was the accuracy of one-step sheet metal forming simulations. In order to validate the new features of SIMEX, we referred to the component shown in Figure 12. RENAULT had designed, built, instrumented and measured this component to reproduce deformation patterns of actual industrial components, even though it not correspond to any actual vehicle component.

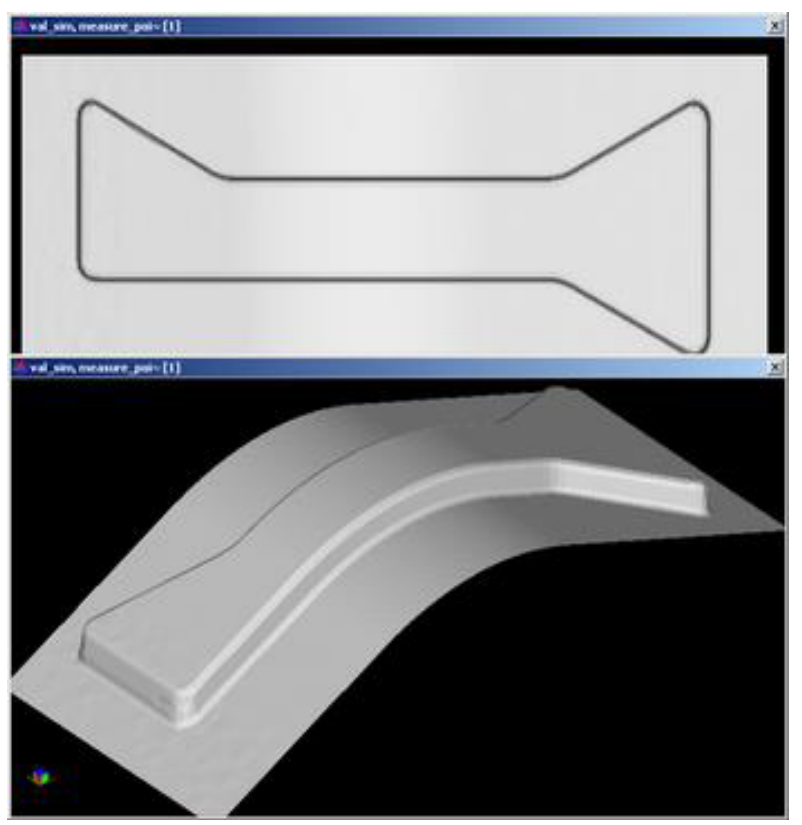

Figure 12 : RENAULT MACARENA rail

The formed material was a steel with the following characteristics:

- thickness $=1.97 \mathrm{~mm}$

- yield strength $=315 \mathrm{Mpa}$

- ultimate strength $=425 \mathrm{Mpa}$

- necking elongation $=18 \%$

- Lankford coefficient $=1.43$

The relevant process data were:

- Blankholder force $=32$ tons

- Friction coeff. $=0.15$

- die entry radius $=3 \mathrm{~mm}$

After forming, measurements of residual thickness have been carried out on 55 points on the part surface $(5$ replications). 17 points were situated along a section at part center, whereas the other 38 points were spread in significant positions over the part surface (Figure 13).

The mesh chosen for simulation (Figure 14) is representative of coupling with crash, fatigue or other engineering analyses. It consists of a total of 5321 elements (4034 trias, 1287 quads).

The mesh is divided in three parts, approximatively modeling the forming process:

- A bottom part, sticking to the punch

- A wall part, which flows over the die radius during the forming process
- A blankholder part, subjected to the 32 ton holding force

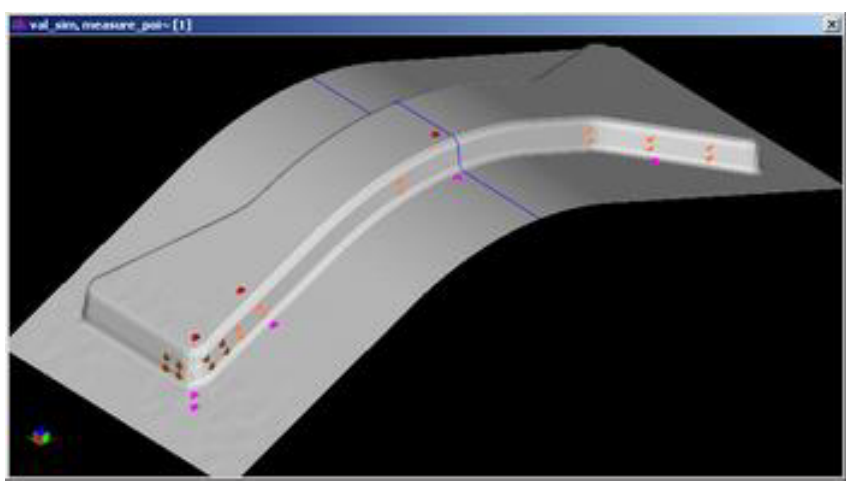

Figure 13: MACARENA rail with measurement points

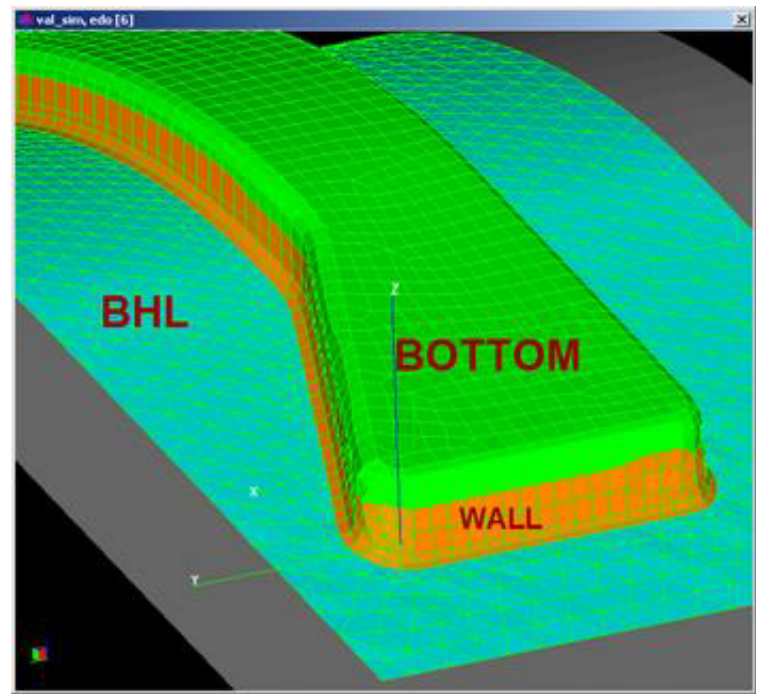

Figure 14: SIMEX mesh for MACARENA rail

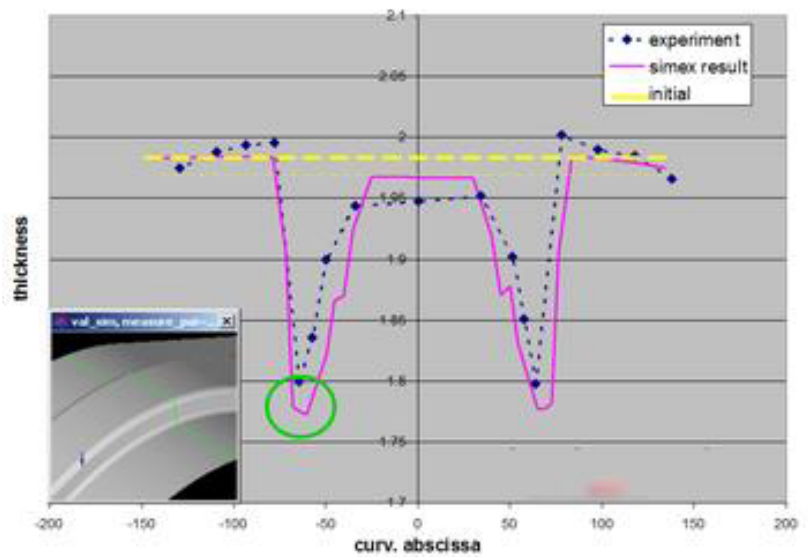

Figure 15: SIMEX vs. measured thickness of central section

Flexure conditions are generated along the die entry radius to simulate the flexure cycle.

Figure 15 presents the comparison of the residual thickness along the central line. SIMEX results are shown with respect to experimental measurements. The qualitative comparison is excellent, The peaks of deformation are correctly reproduced as well as the region of low deformation. As for quantitative results, 
maximum difference between experimental measurement and simulation is $30 \mu \mathrm{m}$.

\section{Conclusions}

Material flow over a die radius and the associated cycles of bending and unbending happen on a relatively small scale, with respect to the average element size in one step simulation. We have developed a semi-analytical model for the prediction of the residual strains and stresses in the sections after the die radius and we have implemented it into SIMEX code.

The application of this model has greatly improved the prediction capabilities of the code. On the industrial parts studied, the agreement between numerical results and SIMEX prediction is excellent.

\section{Acknowledgments}

Part of the work presented has been carried out within the framework of the RNTL MACARENA project. The support of the French Ministry of Industry is dutifully acknowledged.The author would like to thank the RENAULT partcipants to MACARENA project (in particular Mr. Pierre LORY) for their cooperation

\section{References}

[1] Zhou, D., Wagoner, R.H., J. Materials Processing Technology, 50 (1995), 1-16.

[2] Makinouchi, A., J. Materials Processing Technology, 60 (1996), 19-26.

[3] El Mouatassim, M. , Thomas, B., Jameux, J-P. DiPasquale, E., Simulation of Materials Processing : Theory, Methods and Applications, Shen \& Dawson (eds), Balkema, Rotterdam (1995).

[4] Nine, H. D., "New drawbead concepts for sheet metal forming", Journal of Applied Metal Working, Vol. 2, No. 3, July 1982.

[5] Firat, M., Kaftanouglu, B., Eser, O., J. Materials Processing Technology, 196 (2008) 135-148.

[6] Sanchez, L.R., Int. J. of Mechanical Sciences, 53 (2010) 429-439.

[7] Nanu, N., Brabie, G., Int. J. of Mechanical Sciences, 64 (2012) 11-21

[8] Ghaei, A.,Green, D.E., Aryanpour, A., Materials and Design, 88 (2015) 461-470.

[9] Lemaitre, J., Chaboche, J.-L., « Mechanics of solid materials », Cambridge Univ. Press, 1994

[10] Malvern, L.E., "Introduction to the mechanics of a continuous medium”, Prentice-Hall 1969. 